\title{
SPECTRAL SYNTHESIS IN THE KERNEL OF A CONVOLUTION OPERATOR ON WEIGHTED SPACES
}

\author{
R. S. YULMUKHAMETOV
}

\begin{abstract}
Weighted spaces of analytic functions on a bounded convex domain $D \subset \mathbb{C}^{p}$ are treated. Let $U=\left\{u_{n}\right\}_{n=1}^{\infty}$ be a monotone decreasing sequence of convex functions on $D$ such that $u_{n}(z) \longrightarrow \infty$ as $\operatorname{dist}(z, \partial D) \longrightarrow 0$. The symbol $H(D, U)$ stands for the space of all $f \in H(D)$ satisfying $|f(z)| \exp \left(-u_{n}(z)\right) \longrightarrow 0$ as $\operatorname{dist}(z, \partial D) \longrightarrow 0$, for all $n \in \mathbb{N}$. This space is endowed with a locally convex topology with the aid of the seminorms $p_{n}(f)=\sup _{z \in D}|f(z)| \exp \left(-u_{n}(z)\right), n=1,2, \ldots$ Clearly, every functional $S \in H^{*}(D)$ is a continuous linear functional on $H(D, U)$, and the corresponding convolution operator $M_{S}: f \longrightarrow S_{w}(f(z+w))$ acts on $H(D, U)$. All elementary solutions of the equation

$(*)$

$$
M_{S}[f]=0,
$$

i.e., all solutions of the form $z^{\alpha} e^{\langle a, z\rangle}, \alpha \in \mathbb{Z}_{+}^{p}, a \in \mathbb{C}^{p}$, belong to $H(D, U)$. It is shown that the system $E(S)$ of elementary solutions is dense in the space of solutions of equation $(*)$ that belong to $H(D, U)$.
\end{abstract}

\section{INTRODUCTION}

For a domain $D$ in $\mathbb{C}^{p}$, let $H(D)$ denote the space of functions holomorphic in $D$, and let $H^{*}(D)$ be the strong dual of $H(D)$. By the Hahn-Banach theorem, every $S \in H^{*}(D)$ can be extended up to a functional on the space $C(D)$ of continuous functions and can be represented in the form

$$
S(f)=\int_{D} f d \mu, \quad f \in H(D),
$$

where $\mu$ is a measure supported on a compact subset of $D$. Since the support of $\mu$ is compact, we can introduce the following convolution operator generated by $S$ : $M_{S}[f](\zeta)=S_{z}(f(z+\zeta)), f \in H(D)$. The values of $M_{S}$ are functions defined and holomorphic near $\zeta=0$. Let $E(S)$ denote the set of "elementary" solutions of the homogeneous convolution equation

$$
M_{S}[f](\zeta) \equiv 0, \quad f \in H(D)
$$

i.e., $E(S)$ consists of solutions of the form $f(z)=z^{\alpha} \exp \langle z, \xi\rangle$, where $\alpha=\left(\alpha_{1}, \ldots, \alpha_{p}\right)$, $\alpha_{i} \geq 0$, are nonnegative integers, and $\langle z, \xi\rangle=\sum_{i=1}^{p} z_{i} \xi_{i}, z^{\alpha}=z_{1}^{\alpha_{1}} \cdots z_{p}^{\alpha_{p}},|\alpha|$ being smaller than the multiplicity of the root $\xi$. We remind the reader that if $\widehat{S}(\lambda)=$ $S_{z}(\exp \langle\lambda, z\rangle)$ is the Laplace transform of $S$, and the function $f(z)=z^{\alpha} \exp \langle z, \xi\rangle$ is an elementary solution of (2), then $\widehat{S}(\xi)=0$. Denoting by $W(D, S)$ the set of all solutions of equation (2), we ask whether $E(S)$ is dense in the space $W(D, S)$ of all solutions. If the answer is in the positive, the space $W(D, S)$ is said to admit spectral synthesis.

2000 Mathematics Subject Classification. Primary 32A50, 45E10, 46E10.

Key words and phrases. Weighted spaces of analytic functions, convolution operator, spectral synthesis.

Supported by RFBR (grant 06-01-00516-a.) 
In dimension 1, a positive answer was obtained in [2] for convex domains. For some specific types of domains $D \subset \mathbb{C}^{p}$, the completeness of the system of elementary solutions was established in [3, 4, 5]. In [6], the presence of spectral synthesis was announced in the case of arbitrary multidimensional convex domains. A new version of that proof provides a solution of the spectral synthesis problem in weighted spaces of holomorphic functions. We begin with the definition of these spaces.

Let $D$ be a bounded convex domain in $\mathbb{C}^{p}$, and let $U=\left\{u_{n}\right\}_{n=1}^{\infty}$ be a monotone decreasing sequence of convex functions on $D$ such that $u_{n}(z) \longrightarrow \infty$ as $\operatorname{dist}(z, \partial D) \longrightarrow 0$. We denote by $H(D, U)$ the space of functions $f \in H(D)$ satisfying $|f(z)| \exp \left(-u_{n}(z)\right) \longrightarrow$ 0 as $\operatorname{dist}(z, \partial D) \longrightarrow 0$, for all $n \in \mathbb{N}$. A locally convex topology is introduced in this space with the help of the seminorms $p_{n}(f)=\sup _{z \in D}|f(z)| \exp \left(-u_{n}(z)\right), n=1,2, \ldots$ Clearly, an arbitrary $S \in H^{*}(D)$ is a continuous linear functional on $H(D, U)$, and the corresponding convolution operator $M_{S}$ acts on $H(D, U)$. All elementary solutions of equation (2) belong to $H(D, U)$. It is natural to ask whether the system $E(S)$ is dense in the space of all solutions of $(2)$ that belong to $H(D, U)$. In this paper, a positive answer is given under certain conditions on the weights. The proof follows the usual pattern involving the Laplace transformation. For a continuous linear functional $S \in H^{*}(D, U)$, its Laplace transform is defined by $\widehat{S}(\lambda)=S_{z}(\exp \langle\lambda, z\rangle), \lambda \in \mathbb{C}^{p}$. The following theorem was proved in [8].

Theorem A. Let $U=\left\{u_{n}, n \in \mathbb{N}\right\}$ be a monotone decreasing sequence of convex functions on a bounded convex domain $D$, and let $v_{n}(\lambda)=\sup \left\{\operatorname{Re}\langle\lambda, \bar{z}\rangle-u_{n}(z): z \in D\right\}$, $\lambda \in \mathbb{C}^{p}, n \in \mathbb{N}$, be the Young conjuate functions for $u_{n}(z)$. Suppose that the following conditions are satisfied:

1) for every $n$ there exists a number $c_{n}$ with

$$
v_{n+1}(\lambda) \geq v_{n}(\lambda)+\ln \left(1+|\lambda|^{2}\right)+c_{n}, \quad \lambda \in \mathbb{C}^{p} ;
$$

2) for every $n$ we have $v_{n} \in C^{2}\left(\mathbb{C}^{p}\right)$, and there exists $b_{n}>0$ such that

$$
\sum_{k, m=1}^{2 p} \frac{\partial^{2} v_{n}(\lambda)}{\partial x_{k} \partial x_{m}} y_{k} y_{m} \geq \frac{12 p+b_{n}}{1+|\lambda|^{2}}|y|^{2}, \quad \lambda=\left(x_{1}+i x_{2}, \ldots, x_{2 p-1}+i x_{2 p}\right) \in \mathbb{C}^{p}
$$

for all $y=\left(y_{1}, \ldots, y_{2 p}\right) \in \mathbb{R}^{2 p}$.

Let $P\left(v_{n}\right)$ denote the space of entire functions $F$ with the norm

$$
\|F\|_{n}=\sup \left\{|F(\lambda)| \exp \left(-v_{n}(\lambda)\right): \lambda \in \mathbb{C}^{p}\right\},
$$

and let $P(D, U)$ be the union of these spaces with the topology of inductive limit. Under the above assumptions, the Laplace transformation $L: S \longmapsto \widehat{S}$ is a topological isomorphism of the strong dual space $H^{*}(D, U)$ onto $P(D, U)$.

In [7, this theorem was announced without restrictions on the quadratic forms corresponding to $v_{n}$.

For every natural number $m$, put

$$
D_{m}^{\prime}=\left\{z \in D: \operatorname{dist}(z, \partial D)>\frac{1}{m}\right\} .
$$

Let $D_{m}, D_{m}^{\prime} \subset D_{m} \subset D_{m+1}^{\prime}$, be a sequence of convex domains with $C^{2}$-boundary. As in 15] we agree that the functions

$$
u_{m}(z)= \begin{cases}0 & \text { if } z \in \bar{D}_{m} \\ +\infty & \text { if } z \in D \backslash \bar{D}_{m}\end{cases}
$$

are convex. If $U=\left(u_{m}\right)_{m=1}^{\infty}$, then $H(D, U)=H(D)$. It is well known (see [1]) that the Laplace transformation establishes a topological isomorphism between $H^{*}(D)$ and 
the space $P(D)$ of the entire functions $F$ that obey the following condition: there is a compact subset $K$ of $D$ and a constant $C$ such that $|F(\lambda)| \leq C \exp \left(h_{K}(\lambda)\right), \lambda \in \mathbb{C}^{p}$, where $h_{K}(\lambda)=\sup \{\operatorname{Re}\langle\lambda, \bar{z}\rangle: z \in K\}$ is the support function for $K$. The space $P(D)$ admits the following description. Let $h_{m}$ denote the support function for $\bar{D}_{m}$, and let $P\left(h_{m}\right)$ be the Banach space of entire functions with the norm $\|F\|_{m}=\sup \left\{|F(\lambda)| \exp \left(-h_{m}(\lambda)\right)\right.$ : $\left.\lambda \in \mathbb{C}^{p}\right\}$. Then $P(D)$ is the union of all $P\left(h_{m}\right)$ with the topology of inductive limit. Moreover, the Young conjugates to $u_{m}$ are $h_{m}$, and $P(D)=P(D, U)$. So, the Laplace transformation is a topological isomorphism between $H^{*}(D, U)$ and $P(D, U)$; see [1].

Theorem 1. Let $U=\left\{u_{n}, n \in \mathbb{N}\right\}$ be a monotone decreasing sequence of convex functions on a bounded domain D. Suppose that the Laplace transformation is a topological isomorphism between $H^{*}(D, U)$ and $P(D, U)$. If the Young conjugates $v_{n}$ for $u_{n}$ satisfy (3), then, for any $S \in H^{*}(D)$, an arbitrary solution of (2) belonging to $H(D, U)$ can be approximated by linear combinations of elementary solutions in the topology of $H(D, U)$.

The paper is organized as follows.

In $\S 1$, by using methods described in preceding work of various authors, we reduce the spectral synthesis problem in question to the problem of completeness of the polynomials in certain weighted spaces of entire functions.

In $\S 2$, we study this completeness problem itself independently.

Finally, in $\S 3$, we deduce Theorem 1 from the results of $\S \S 1,2$.

\section{§1. Spectral Synthesis and COMPleteness of POlynomials}

Let $U=\left\{u_{n}, n \in \mathbb{N}\right\}$ be a monotone decreasing sequence of convex functions defined on a bounded convex domain $D$ and satisfying the assumptions of Theorem 1 . As usual, $X^{\perp}$ stands for the annihilator of a subset $X$ of a linear topological space.

For $S \in H^{*}(D)$, we denote by $W(D, U, S)$ the set of solutions of equation (2) in the space $H(D, U)$. By the Banach theorem, spectral synthesis for $W(D, U, S)$ is equivalent to the identification of the annihilators $E^{\perp}(S)$ and $W^{\perp}(D, U, S)$. We need the following properties of the space $H(D, U)$.

Lemma 1. Under the assumptions of Theorem 1, we have the following statements.

1. $H(D, U)$ is a complete locally convex reflexive space, i.e., $\left(H^{*}(D, U)\right)^{*}=H(D, U)$.

2. Differentiation operators act continuously on $H(D, U)$.

3. Putting $J(D, U, S)=\left\{f \in P(D, U): f / \widehat{S} \in H\left(\mathbb{C}^{p}\right)\right\}$, we have $L\left(E^{\perp}(S)\right)=$ $J(D, U, S)$.

4. Denoting by $I(D, U, S)$ the closure of $I^{\prime}(S)=\{f \in P(D, U): f / \widehat{S}$ is a polynomial $\}$ in $P(D, U)$, we have $L\left(W^{\perp}(D, U, S)\right)=I(S)$.

5. The elementary solutions are complete in the space of solutions of equation (2) if and only if $I(D, U, S)=J(D, U, S)$.

Proof. The first statement can be proved by standard methods on the basis of results of 9.

By (3), the operator of multiplication by $\lambda_{1}$ (say) acts continuously on $P(D, U)$. Under the Laplace transformation isomorphism, multiplication by $\lambda_{1}$ turns into a continuous operator $A$ on $H^{*}(D, U)$, specifically, the operator of "convolution multiplication" by the functional $\delta^{\prime}, \delta^{\prime}(f)=\frac{\partial f}{\partial z_{1}}(0), f \in H(D, U)$. By statement $1, A^{*}$ acts continuously on $H(D, U)$, and it can easily be shown (see [14]) that $A^{*}$ coincides with differentiation by the variable $\lambda_{1}$.

Statements 3 and 4 are proved much as the corresponding statements for $H(D)$; see 10. We have already established the prerequisites, i.e., the reflexivity of $H(D, U)$ and its 
invariance under differentiation (the latter is a consequence of the Banach completeness theorem).

Restated in more detail, the last statement of the lemma means that spectral synthesis occurs in the kernel of a convolution operator on $H(D, U)$ if and only if every function of the form $f \widehat{S}$ and belonging to $P(D, U)$ can be approximated by functions of the form $p \widehat{S}$, where $p$ is a polynomial. Taking the topology of $P(D, U)$ into account, we can formulate this condition as follows: for every $f \widehat{S} \in P(D, U)$, there exists $m \in \mathbb{N}$ and a sequence $p_{n}$ of polynomials such that

$$
\left|f(\lambda)-p_{n}(\lambda)\right| \leq \varepsilon_{n} \frac{e^{v_{m}(\lambda)}}{|\widehat{S}(\lambda)|}, \quad \lambda \in \mathbb{C}^{p},
$$

where $\varepsilon_{n} \longrightarrow 0$ as $n \longrightarrow \infty$. Thus, we arrive at the problem of completeness of the polynomials in a certain weighted space of entire functions.

\section{§2. Completeness of the polynomials in Weighted SPACES OF ENTIRE FUNCTIONS}

For a plurisubharmonic (p.s.h.) function $v$ on $\mathbb{C}^{p}$, we denote by $P(v)$ the space of entire functions with the norm $\|F\|_{P(v)}:=\sup _{\lambda \in \mathbb{C}^{p}}(|F(\lambda)| \exp (-v(\lambda)))$, and by $P_{2}(v)$ the Hilbert space of entire functions with the norm $\|F\|_{P_{2}(v)}^{2}:=\int_{\mathbb{C}^{p}}|F(\lambda)|^{2} \exp (-2 v(\lambda)) d m(\lambda)$. Let $D(z, r)$ be the ball in $\mathbb{C}^{p}$ centered at $z$ and of radius $r$. In what follows, we often impose the following condition on a p.s.h. function:

$$
|\varphi(\lambda)-\varphi(\zeta)| \leq B, \quad \zeta \in D\left(\lambda,\left(1+|\lambda|^{2}\right)^{-s}\right), \quad \lambda \in \mathbb{C}^{p} .
$$

(Then we say that $\varphi$ satisfies condition (R) with constants $B, s>0$.) The following theorem is the main result of this section.

Theorem 2. Let $v(\lambda)$ be a p.s.h. function on $\mathbb{C}^{p}$ satisfying $(\mathrm{R})$. Suppose also that there exist constants $\delta, \Delta>0$ such that

$$
\delta(1+|\lambda|) \leq v(\lambda) \leq \Delta(1+|\lambda|), \quad \lambda \in \mathbb{C}^{p} .
$$

Then every function in $P(v)$ can be approximated by polynomials in the norm of $P\left(v+(p s+p+1) \ln \left(1+|\lambda|^{2}\right)\right)$.

In essence, the proof is based on a theorem in [11, Lemma 2.2 in 10, and the Hörmander theorem on $L_{2}$-estimates. We start with formulating these statements in a form suitable for the present setting.

Theorem B (see [11]). Put $\varphi(\lambda)=\sup ^{*} \varphi_{\alpha}(\lambda), \lambda \in \mathbb{C}^{p}$, where $\varphi_{\alpha}$ is a family of p.s.h. functions on $\mathbb{C}^{p}$ that filters to the right. Then the functions in $P_{2}(\varphi)$ can be approximated by functions in $\bigcup_{\alpha} P_{2}\left(\varphi_{\alpha}\right)$ in the norm of $P_{2}\left(\varphi+\ln \left(1+|\lambda|^{2}\right)\right)$. In particular, if the $\varphi_{\alpha}$ are of logarithmic growth, i.e., $\varphi_{\alpha}(\lambda)=O(\ln |\lambda|)$ as $\lambda \longrightarrow \infty$, we deal with approximation by polynomials.

Lemma C (see [10, Lemma 12.2]). Suppose $\varphi$ is a p.s.h. function on $\mathbb{C}^{p}$, and for some $\varepsilon>0$ a function $f \in P(\varphi+\varepsilon|\lambda|)$ is the product of some functions $f_{k} \in P\left(\frac{1}{N}(\varphi+\varepsilon|\lambda|)\right)$, $k=1,2, \ldots, N$. If $\frac{1}{N}(\varphi(\lambda)+\varepsilon|\lambda|) \leq C+\varepsilon|\lambda|, \lambda \in \mathbb{C}^{p}$, then $f$ can be approximated by polynomials in the norm of the space $P(\varphi+4 \varepsilon|\lambda|)$.

It should be noted that the spectral synthesis problem under study will be reduced to the problem of weighted uniform approximation by polynomials, whereas we are going to employ Theorem B and Hörmander's $L_{2}$-estimates, where weighted integral norms are involved. For this reason, we explain how to pass to weighted integral norms from weighted uniform norms and vice versa. 
Lemma 2. 1. If $\varphi$ is a p.s.h. function on $\mathbb{C}^{p}$ and $F \in P(\varphi)$, then

$$
\|F\|_{P_{2}\left(\varphi+p \ln \left(1+|\lambda|^{2}\right)\right)} \leq \sqrt{2 S_{p}}\|F\|_{P(\varphi)} .
$$

2. If a p.s.h. function $\varphi$ on $\mathbb{C}^{p}$ satisfies $(\mathrm{R})$, and $F \in P_{2}(\varphi)$, then

$$
\|F\|_{P\left(\varphi+p s \ln \left(1+|\lambda|^{2}\right)\right)} \leq \frac{e^{B}}{\sqrt{V}_{p}}\|F\|_{P_{2}(\varphi)} .
$$

Here $S_{p}$ and $V_{p}$ are the area of the unit sphere and the volume of the unit ball in $\mathbb{C}^{p}$.

Proof. Statement 1 is trivial. The other estimate is also simple: it can be deduced from the fact that $|F|^{2}$ is subharmonic.

Lemma 3. Let $\varphi$ be a p.s.h. function on $\mathbb{C}^{p}$ satisfying $(\mathrm{R})$. Then for every $a \in \mathbb{C}^{p}$ there exist an entire function $F_{a}$ such that $F_{a}(a)=\exp \varphi(a)$ and

$$
\int_{\mathbb{C}^{p}}\left|F_{a}(\lambda)\right|^{2} e^{-2 \varphi(\lambda)-(2 s p+3 p+1) \ln \left(1+|\lambda|^{2}\right)} d m(\lambda) \leq C_{p},
$$

where $C_{p}=\frac{3^{2 s+2}}{1+4 s^{2}}\left(6 e^{2 B} \pi\left(1+4 s^{2}\right)\right)^{p}$.

Proof. We proceed by induction on the dimension of the space. For $p=1$, we put

$$
\alpha(t)= \begin{cases}0 & \text { if }|t| \geq 1 \\ 1 & \text { if }|t| \leq \frac{1}{2} \\ 2(1-|t|) & \text { if } \frac{1}{2} \leq|t| \leq 1\end{cases}
$$

Then $|\partial \alpha(t) / \partial \bar{t}| \leq 1$. Let $a \in \mathbb{C}$ be arbitrary. We define

$$
g(z)=\frac{e^{\varphi(a)}}{(z-a)} \frac{\partial}{\partial \bar{z}} \alpha\left((z-a)\left(1+|a|^{2}\right)^{s}\right), \quad z \in \mathbb{C},
$$

and consider the equation

$$
\frac{\partial}{\partial \bar{z}} u(z)=g(z)
$$

By the properties of $\alpha(z)$ and the conditions imposed on $\varphi(z)$, we have

$$
\int_{\mathbb{C}}|g(z)|^{2} e^{-2 \varphi(z)} d m(z) \leq 4 \pi e^{2 B}\left(1+|a|^{2}\right)^{2 s} .
$$

The elementary estimate

$$
\sup _{|z-\lambda| \leq 1} \frac{1+|z|^{2}}{1+|\lambda|^{2}} \leq \frac{1+(1+|\lambda|)^{2}}{1+|\lambda|^{2}} \leq \frac{3+2|\lambda|^{2}}{1+|\lambda|^{2}} \leq 3
$$

shows that

$$
\int_{\mathbb{C}}|g(z)|^{2} e^{-2 \varphi(z)-(2 s+1) \ln \left(1+|z|^{2}\right)} d m(z) \leq 4 \pi 3^{2 s+1} e^{2 B}\left(1+|a|^{2}\right)^{-1} .
$$

By the Hörmander theorem, equation (2.1) has a solution $u$ satisfying

$$
\int_{\mathbb{C}}|u(z)|^{2} e^{-2 \varphi(z)-(2 s+3) \ln \left(1+|z|^{2}\right)} d m(z) \leq 2 \pi 3^{2 s+1} e^{2 B}\left(1+|a|^{2}\right)^{-1} .
$$

Since $|z-a|^{2} \leq(1+|z|)^{2}(1+|a|)^{2} \leq 4\left(1+|a|^{2}\right)\left(1+|z|^{2}\right)$, we see that

$$
\int_{\mathbb{C}}|z-a|^{2}|u(z)|^{2} e^{-2 \varphi(z)-(2 s+4) \ln \left(1+|z|^{2}\right)} d m(z) \leq 8 \pi 3^{2 s+1} e^{2 B} .
$$


By the definition of $u$, the function $F_{a}(z)=\alpha\left((z-a)\left(1+|a|^{2}\right)^{s}\right) \exp \varphi(a)-(z-a) u(z)$, $z \in \mathbb{C}$, is entire and $F_{a}(a)=\exp \varphi(a)$. Moreover,

$$
\int_{\mathbb{C}}\left|F_{a}(z)\right|^{2} e^{-2 \varphi(z)-(2 s+4) \ln \left(1+|z|^{2}\right)} d m(z) \leq 50 \pi 3^{2 s} e^{2 B} .
$$

Suppose the claim is proved for $\mathbb{C}^{p-1}$. Take $a \in \mathbb{C}^{p}$. There is no loss of generality in assuming that $a$ belongs to the $(p-1)$-dimensional subspace $\left\{z_{1}=0\right\}$. By the inductive hypothesis, there exists an entire function $f$ of $z^{\prime}=\left(z_{2}, \ldots, z_{p}\right) \in \mathbb{C}^{p-1}$ such that $f\left(a^{\prime}\right)=e^{\varphi\left(a^{\prime}\right)}, a^{\prime}=\left(a_{2}, \ldots, a_{p}\right)$, and

$$
\int_{\mathbb{C}^{p-1}}|f(z)|^{2} e^{-2 \varphi(z)-(2 s(p-1)+3(p-1)+1) \ln \left(1+|z|^{2}\right)} d m(z) \leq C_{p-1} .
$$

The expression $\varphi\left(a^{\prime}\right)$ is understood as the value of $\varphi$ at $\left(0, a^{\prime}\right) \in \mathbb{C}^{p}$. Put $g(z)=$ $f(z) z_{1}^{-1} \bar{\partial} \alpha\left(z_{1}\left(1+\left|z^{\prime}\right|^{2}\right)^{s}\right), z=\left(z_{1}, \ldots, z_{p}\right) \in \mathbb{C}^{p}$, where $f$ is viewed as a function on $\mathbb{C}^{p}$ depending only on $z^{\prime}=\left(z_{2}, \ldots, z_{p}\right)$, and $\alpha(w), w \in \mathbb{C}$, has already been defined. Direct inspection shows that $\left|\bar{\partial} \alpha\left(z_{1}\left(1+\left|z^{\prime}\right|^{2}\right)^{s}\right)\right|^{2} \leq\left(1+4 s^{2}\right)\left(1+\left|z^{\prime}\right|^{2}\right)^{2 s}$. The properties of $\alpha(w)$ imply $|g(z)|^{2} \leq 4\left|f\left(z^{\prime}\right)\right|^{2}\left(1+4 s^{2}\right)\left(1+\left|z^{\prime}\right|^{2}\right)^{4 s}$; moreover, $g(z)=0$ if $\left|z_{1}\right| \geq\left(1+\left|z^{\prime}\right|^{2}\right)^{-s}$. Thus,

$$
\begin{aligned}
\int_{\mathbb{C}^{p}}|g(z)|^{2} e^{-2 \varphi(z)-(2 s p+3 p-2) \ln \left(1+|z|^{2}\right)} d m(z) & \\
\leq 4\left(1+4 s^{2}\right) & \int_{\mathbb{C}^{p-1}\left(z^{\prime}\right)}\left|f\left(z^{\prime}\right)\right|^{2}\left(1+\left|z^{\prime}\right|^{2}\right)^{4 s} e^{-2 \varphi\left(z^{\prime}\right)-(2 s p+3 p-2) \ln \left(1+\left|z^{\prime}\right|^{2}\right)} \\
& \times \int_{\left|z_{1}\right| \leq\left(1+\left|z^{\prime}\right|^{2}\right)^{-s}} e^{2\left(\varphi\left(z^{\prime}\right)-\varphi(z)\right)+(2 s p+3 p-2) \ln \frac{1+\left|z^{\prime}\right|^{2}}{1+|z|^{2}}} d m\left(z_{1}\right) d m\left(z^{\prime}\right) .
\end{aligned}
$$

Condition (R) for $\varphi$ shows that

$$
\int_{\mathbb{C}^{p}}|g(z)|^{2} e^{-2 \varphi(z)-(2 s p+3 p-2) \ln \left(1+|z|^{2}\right)} d m(z) \leq 4 \pi\left(1+4 s^{2}\right) e^{2 B} C_{p-1} .
$$

Now we consider the equation $\bar{\partial} u=g$. By the Hörmander theorem, this equation has a solution $u$ with

$$
\int_{\mathbb{C}^{p}}|u(z)|^{2} e^{-2 \varphi(z)-(2 s p+3 p) \ln \left(1+|z|^{2}\right)} d m(z) \leq 2 \pi\left(1+4 s^{2}\right) e^{2 B} C_{p-1} .
$$

Then

$$
\int_{\mathbb{C}^{p}}\left|z_{1} u(z)\right|^{2} e^{-2 \varphi(z)-(2 s p+3 p+1) \ln \left(1+|z|^{2}\right)} d m(z) \leq 2 \pi\left(1+4 s^{2}\right) e^{2 B} C_{p-1} .
$$

We put $F_{a}(z)=f\left(z^{\prime}\right) \alpha\left(z_{1}\left(1+\left|z^{\prime}\right|^{2}\right)^{s}\right)-z_{1} u(z), z \in \mathbb{C}^{p}$. The definition of $u$ implies that $F_{a}$ is an entire function. By the inductive hypothesis, $F_{a}(a)=\exp \varphi(a)$. Next, from the properties of $\alpha$ we deduce that

$$
\int_{\mathbb{C}^{p}}\left|f\left(z^{\prime}\right)\right|^{2} \alpha\left(z_{1}\left(1+\left|z^{\prime}\right|^{2}\right)^{s}\right) e^{-2 \varphi(z)-(2 s p+3 p+1) \ln \left(1+|z|^{2}\right)} d m(z) \leq \pi e^{2 B} C_{p-1} .
$$

Together with (2.2) and the triangle inequality for norms, this estimate yields

$$
\int_{\mathbb{C}^{p}}\left|F_{a}(z)\right|^{2} e^{-2 \varphi(z)-(2 s p+3 p+1) \ln \left(1+|z|^{2}\right)} d m(z) \leq e^{2 B} 6 \pi\left(1+4 s^{2}\right) C_{p-1} .
$$

Therefore, we can take $C_{1}=50 \pi e^{2 B} 3^{2 s}, C_{p}=6 \pi e^{2 B}\left(1+4 s^{2}\right) C_{p-1}, p>1$.

Lemma 3 is proved. 
Corollary. Let $\varphi$ be a p.s.h. function on $\mathbb{C}^{p}$ satisfying $(\mathrm{R})$. Then the function $F_{a}$ in Lemma 3 obeys the uniform estimate

$$
\left|F_{a}(\lambda)\right|^{2} \leq c_{p} e^{2 \varphi(\lambda)+(3 s p+3 p+1) \ln \left(1+|\lambda|^{2}\right)}, \quad \lambda \in \mathbb{C}^{p},
$$

where $c_{p}=\frac{e^{2 B} 3^{2 s+2}}{V_{p}\left(1+4 s^{2}\right)}\left(6 \pi e^{2 B}\left(1+4 s^{2}\right)\right)^{p}$.

Proof. This is an immediate consequence of statement 2 in Lemma 2.

Lemma 4. Suppose that $\varphi$ is a nonnegative p.s.h. function on $\mathbb{C}^{p}$ satisfying $(\mathrm{R})$ and such that $\varphi(\lambda) \leq \Delta(1+|\lambda|), \lambda \in \mathbb{C}^{p}$. Then for every $\sigma>0$ the functions in the space $P(\varphi+\sigma|\lambda|)$ admit approximation by polynomials in the norm of $P(\varphi+9 \sigma|\lambda|)$.

Proof. We introduce the following function of the variable $\lambda$ :

$$
w(\lambda)=\sup ^{*}\left\{u(\lambda): u \text { is p.s.h., } u(z)=O(\ln |z|), u(z) \leq \varphi(z)+8 \sigma|z|, z \in \mathbb{C}^{p}\right\} .
$$

Put $N=[\Delta / \sigma]+3$, where $[x]$ denotes the integral part of $x$. Then $(\Delta+2 \sigma) / N \leq \sigma$. The function $u(\lambda)=(\varphi(\lambda)+\sigma|\lambda|) / N$ satisfies condition $(\mathrm{R})$ with constants $(B+\sigma) / N$ and $s$. By the corollary to Lemma 3 , for every $a \in \mathbb{C}^{p}$ there exists a function $F_{a}$ that equals $\exp ((\varphi(a)+\sigma|a|) / N)$ at $a$ and satisfies

$$
\left|F_{a}(\lambda)\right| \leq c_{p} e^{\frac{1}{N}(\varphi(\lambda)+\sigma|\lambda|)+(3 s p+3 p+1) \ln \left(1+|\lambda|^{2}\right)}, \quad \lambda \in \mathbb{C}^{p},
$$

where $c_{p}$ is defined as in the corollary to Lemma 3 and depends only on the dimension $p$ and the numbers $B, s, \Delta, \sigma$. We write the upper estimate for $F_{a}$ in the form $\left|F_{a}(\lambda)\right| \leq c_{p}^{\prime} \exp \left(\frac{1}{N}(\varphi(\lambda)+2 \sigma|\lambda|)\right)$, where $c_{p}^{\prime}$ depends only on $p, B, \Delta, s, \sigma$, and $N$. The function $f_{a}=\left(F_{a} / c_{p}^{\prime}\right)^{N}$ equals $\left(c_{p}^{\prime}\right)^{-N} e^{\varphi(a)+\sigma|a|}$ at $a$ and satisfies the inequality $\left|f_{a}(\lambda)\right| \leq \exp (\varphi(\lambda)+2 \sigma|\lambda|), \lambda \in \mathbb{C}^{p}$. By the choice of $N$, the functions $f_{a}$ satisfy the assumptions of Lemma $\mathrm{C}$ with $2 \sigma$ in the role of $\varepsilon$. That lemma shows that each $f_{a}$ can be approximated by polynomials in the norm of the space $P(\varphi+8 \sigma|\lambda|)$; i.e., for every $\varepsilon>0$ there exists a polynomial $p_{a, \varepsilon}$ with $\left|f_{a}(\lambda)-p_{a, \varepsilon}(\lambda)\right| \leq \varepsilon \exp (\varphi(\lambda)+8 \sigma|\lambda|), \lambda \in \mathbb{C}^{p}$. Then the polynomial $q_{a, \varepsilon}=\frac{1}{1+\varepsilon} p_{a, \varepsilon}$ obeys the inequalities $\left|q_{a, \varepsilon}(\lambda)\right| \leq \exp (\varphi(\lambda)+8 \sigma|\lambda|)$ for all $\lambda$, and $\left|q_{a, \varepsilon}(a)\right| \geq\left(\left(c_{p}^{\prime}\right)^{-N} \exp (\varphi(a)+\sigma|a|)-\varepsilon \exp (\varphi(a)+8 \sigma|a|)\right) /(1+\varepsilon)$. The upper estimate and the definition of $w$ show that $w(\lambda) \geq \ln \left|q_{a, \varepsilon}(\lambda)\right|, \lambda \in \mathbb{C}^{p}$. At the point $a$ we use the lower estimate for $q_{a, \varepsilon}(a)$ and let $\varepsilon$ tend to zero, obtaining $w(a) \geq \varphi(a)+\sigma|a|-N \ln c_{p}^{\prime}$, $a \in \mathbb{C}^{p}$. It follows that the space $P(\varphi+\sigma|\lambda|)$ is included in $P(w)$. By statement 1 of Lemma 2, we have $P(w) \subset P_{2}\left(w+p \ln \left(1+|\lambda|^{2}\right)\right)$. By the definition of $w$, the function $w+p \ln \left(1+|\lambda|^{2}\right)$ is the upper envelope of a family of p.s.h. functions of logarithmic growth.

By Theorem $\mathrm{B}$, the functions belonging to $P_{2}\left(w+p \ln \left(1+|\lambda|^{2}\right)\right.$ ) (and a fortiori those belonging to $P(\varphi+\sigma|\lambda|))$ can be approximated by polynomials in the norm of $P_{2}\left(w+(p+1) \ln \left(1+|\lambda|^{2}\right)\right)$, consequently, also in the norm of $P_{2}(\varphi+8 \sigma|\lambda|)$. Since we can replace $\varphi$ by $\varphi+8 \sigma|\lambda|$ in statement 2 of Lemma 2, the latter function satisfies condition (R) with the constants $B+8 \sigma$ and $s$. Taking statement 2 of Lemma 2 into account, we see that the functions belonging to $P(\varphi+\sigma|\lambda|)$ can be approximated by polynomials in the norm of $P\left(\varphi+8 \sigma|\lambda|+p s \ln \left(1+|\lambda|^{2}\right)\right)$, consequently, in the norm of $P(\varphi+9 \sigma|\lambda|)$. This finishes the proof of Lemma 4 .

Proof of Theorem 2. Suppose a function $v$ satisfies the assumptions of Theorem 2. Then so does also the function $v_{1}(\lambda)=v(\lambda)+p \ln \left(1+|\lambda|^{2}\right)$ with the constants $B+p \ln 3$ and $s$ in condition $(\mathrm{R})$ and with $\Delta+p$ in place of $\Delta$. For $\alpha \in(0 ; 1)$, we denote the function $(1-\alpha) v_{1}$ by $\varphi_{\alpha}$, and apply Lemma 4 with $\sigma_{\alpha}=\frac{\alpha \delta}{10}$ to it. This shows that all functions belonging to $P\left(\varphi_{\alpha}+\sigma_{\alpha}|\lambda|\right)$ can be approximated by polynomials in the norm of 
$P\left(\varphi_{\alpha}+9 \sigma_{\alpha}|\lambda|\right)$. Since $v_{1}(\lambda) \geq v(\lambda) \geq \delta(1+|\lambda|) \geq \delta|\lambda|$ and $\varphi_{\alpha}(\lambda)+9 \sigma_{\alpha}|\lambda|+p \ln \left(1+|\lambda|^{2}\right) \leq$ $v_{1}(\lambda)-\frac{\alpha \delta}{10}|\lambda|+p \ln \left(1+|\lambda|^{2}\right) \leq v_{1}(\lambda)+$ const for all $\lambda$, statement 1 of Lemma 2 implies that for any $\alpha \in(0 ; 1)$ the functions in $P\left(\varphi_{\alpha}+\sigma_{\alpha}|\lambda|\right)$ can be approximated by polynomials in the norm of $P_{2}\left(v_{1}\right)$. The function $\varphi_{\alpha}+\sigma_{\alpha}|\lambda|$ satisfies condition (R) with the constants $(1-\alpha)(B+p \ln 3) \sigma_{\alpha}$ and $s$. By Lemma 2 ,

$$
\bigcup_{\alpha \in(0 ; 1)} P\left(\varphi_{\alpha}+\sigma_{\alpha}|\lambda|\right)=\bigcup_{\alpha \in(0 ; 1)} P_{2}\left(\varphi_{\alpha}+\sigma_{\alpha}|\lambda|\right) .
$$

Thus, the functions in these unions can be approximated by polynomials in the norm of $P_{2}\left(v_{1}\right)$. Since $\varphi_{\alpha}(\lambda)+\sigma_{\alpha}|\lambda| \leq v_{1}(\lambda)$ and $\lim _{\alpha \rightarrow 0}\left(\varphi_{\alpha}(\lambda)+\sigma_{\alpha}|\lambda|\right)=v_{1}(\lambda)$ for every $\lambda \in \mathbb{C}^{p}$, we see that $\sup ^{*}\left(\varphi_{\alpha}(\lambda)+\sigma_{\alpha}|\lambda|\right)=v_{1}(\lambda)$. By Theorem B, the functions belonging to $P_{2}\left(v_{1}\right)$ can be approximated by functions belonging to $\bigcup_{\alpha \in(0 ; 1)} P_{2}\left(\varphi_{\alpha}+\sigma_{\alpha}|\lambda|\right)$ in the norm of $P_{2}\left(v_{1}+\ln \left(1+|\lambda|^{2}\right)\right)$. Hence, the functions in $P_{2}\left(v_{1}\right)$ can be approximated by polynomials in the norm of $P_{2}\left(v_{1}(\lambda)+\ln \left(1+|\lambda|^{2}\right)\right)$. Since the function $v_{1}(\lambda)+$ $\ln \left(1+|\lambda|^{2}\right)=v(\lambda)+(p+1) \ln \left(1+|\lambda|^{2}\right)$ satisfied condition $(\mathrm{R})$, statement 2 of Lemma 2 shows that the functions in $P(v)$ can be approximated by polynomials in the norm of $P\left(v+(p s+p+1) \ln \left(1+|\lambda|^{2}\right)\right)$. The proof of Theorem 2 is complete.

\section{§3. Proof of Theorem 1}

In $\S 1$, we reduced the spectral synthesis problem in the kernel of the convolution operator $M_{S}, S \in H^{*}(D)$, to the question of completeness of the polynomials in the inductive limit of the weighted spaces $P\left(v_{m}-\ln |\widehat{S}|\right)$. We start with a description of these spaces in terms of weights more suitable for an application of Theorem 2. We formulate two simple statements.

Lemma 5. Suppose $u$ is a lower semicontinuous function on $\bar{D}$ and denote by $v(\lambda)=$ $\sup \{\operatorname{Re}\langle\lambda, z\rangle-u(z): z \in D\}$ the Young conjugate to $u$. Then $v$ satisfies the Lipschitz condition $\left|v\left(\lambda_{1}\right)-v\left(\lambda_{2}\right)\right| \leq M(D)\left|\lambda_{1}-\lambda_{2}\right|, \lambda_{1}, \lambda_{2} \in \mathbb{C}^{p}$, where $M(D)=\max \{|z|, z \in \bar{D}\}$.

This is a direct consequence of the definition of the Young conjugate function.

Lemma 6. Let $w$ and $v$ be subharmonic functions on $\mathbb{R}^{m}$ satisfying $w(0)>-\infty$ and $v(x)+w(x) \leq C(1+|x|), w(x) \leq c(1+|x|), x \in \mathbb{R}^{m}$. Then

$$
v(x) \leq\left(4 C+2^{m+1} c\right)(1+|x|)-2^{m} w(0), \quad x \in \mathbb{R}^{m} .
$$

Proof. In dimension $2(m=2)$, the claim follows from a lemma in 13. For $m>2$ the proof is similar.

For every $m \in \mathbb{N}$, we introduce the following families of functions:

$$
\mathrm{K}_{m}=\left\{t: t \text { is p.s.h. in } \mathbb{C}^{p}, t(\lambda) \leq v_{m}(\lambda)-\ln |\widehat{S}(\lambda)|, \lambda \in \mathbb{C}^{p}\right\} .
$$

Since the determining set of the functional $S$ is compact, the family $\mathrm{K}_{m}$ is nonempty for $m$ sufficiently large. Each of these families is upper bounded. Indeed, take an infinitely differentiable nonnegative function $\alpha$ depending only on $|z|$, equal to 0 for $|z|>1$ and with integral 1 . For every locally integrable function $f$, we introduce its regularization

$$
f_{\varepsilon}(z)=\int_{\mathbb{C}^{p}} \frac{1}{\varepsilon^{2 p}} \alpha\left(\frac{\zeta-z}{\varepsilon}\right) f(\zeta) d m(\zeta)
$$

where $\varepsilon>0$ and integration is with respect to Lebesgue measure in $\mathbb{C}^{p}$. It is known (see [12]) that the regularizations are infinitely differentiable and, if $f$ is (pluri)subharmonic, then $f_{\varepsilon}$ is also (pluri)subharmonic and $f_{\varepsilon}(z) \geq f(z)$ for every $\varepsilon>0$. Take $\varepsilon=1$. Since the regularization $\left(v_{m}-\ln |\widehat{S}|\right)_{\varepsilon}$ is continuous, it is bounded on each compact set. The properties of regularizations of p.s.h. functions show that for every $t \in \mathrm{K}_{m}$ and all $\lambda$ 
we have $t(\lambda) \leq t_{\varepsilon}(\lambda) \leq\left(v_{m}-\ln |\widehat{S}|\right)_{\varepsilon}(\lambda)$. So, the upper envelope $V_{m}(\lambda)=\sup ^{*}\{t(\lambda)$ : $\left.t \in \mathrm{K}_{m}\right\}$ is a p.s.h. function. If $F$ is entire and $\ln |F(\lambda)| \leq v_{m}(\lambda)-\ln |\widehat{S}(\lambda)|$ for all $\lambda \in \mathbb{C}^{p}$, then $\ln |F| \in \mathrm{K}_{m}$. By the definition of $V_{m}$, we obtain $\ln |F(\lambda)| \leq V_{m}(\lambda)$ for all $\lambda$. Consequently,

$$
\sup _{\mathbb{C}^{p}}|F(\lambda)| e^{-V_{m}(\lambda)}=\sup _{\mathbb{C}^{p}}|F(\lambda)| \frac{|\widehat{S}(\lambda)|}{e^{v_{m}(\lambda)}}, \quad \lambda \in \mathbb{C}^{p} .
$$

Lemma 7. Suppose a functional $S$ admits a representation in the form (1), and $u$ is a lower semicontinuous function on $D$ bounded from above on some set $D_{m}$ (see (4)) containing the support of $\mu$. Put $v(\lambda)=\sup \{\operatorname{Re}\langle\lambda, z\rangle-u(z): z \in D\}$,

$$
\begin{aligned}
\mathrm{K} & =\left\{t: t \text { is p.s.h. in } \mathbb{C}^{p}, t(\lambda) \leq v(\lambda)-\ln |\widehat{S}(\lambda)|, \lambda \in \mathbb{C}^{p}\right\}, \\
V(\lambda) & =\sup ^{*}\{t(\lambda): t \in \mathrm{K}\}, \quad w(z)=\int_{\mathbb{C}^{p}} \alpha(\zeta-z) V(\zeta) d m(\zeta) .
\end{aligned}
$$

Then the following statements hold true:

1. $V(\lambda) \leq w(\lambda) \leq V(\lambda)+M(D), \lambda \in \mathbb{C}^{p}, M(D)=\sup \{|z|: z \in D\}$.

2. There exists a constant $B$ depending only on $D, S$, and $u$ such that $|\operatorname{grad} w(\lambda)| \leq$ $B \sqrt{|\lambda|^{2}+1}, \lambda \in \mathbb{C}^{p}$.

Proof. The left inequality in item 1 is a direct consequence of the properties of regularization for p.s.h. functions. Next, the definition of $V$ implies that

$$
w(z) \leq \int_{\mathbb{C}^{p}} \alpha(\zeta-z)(v(\zeta)-\ln |\widehat{S}(\zeta)|) d m(\zeta) .
$$

Since the function $\ln |\widehat{S}(\zeta)|$ is subharmonic and the function $v$ is Lipschitzian (Lemma 5), we have

$$
w(z) \leq v(z)+M(D)-\ln |\widehat{S}(z)| .
$$

This means that the function $w(z)-M(D)$ belongs to $\mathrm{K}$, and the right inequality in item 1 follows.

Now, we estimate the gradient of $w$. Let $M=\sup |\operatorname{grad} \alpha(z)|$. Then

$$
|\operatorname{grad} w(\lambda)| \leq M \int_{D(\lambda, 1)}|V(z)| d m(z), \quad \lambda \in \mathbb{C}^{p},
$$

and we must estimate the integral of $|V|$ over the ball $D(\lambda, 1)$ in $\mathbb{C}^{p}$. For this, we apply Lemma 6 to the functions $V$ and $\ln |\widehat{S}|$. By the assumptions of that lemma, the functional $S$ is representable in the form (1) and $\operatorname{supp}(\mu) \subset D_{m}$; therefore,

$$
|\widehat{S}(\lambda)| \leq|\mu|(D) \text { exp } \sup _{z \in D_{m}} \operatorname{Re}\langle\lambda, z\rangle=|\mu|(D) e^{h_{m}(\lambda)}, \quad \lambda \in \mathbb{C}^{p} .
$$

Consequently,

$$
\ln |\widehat{S}(\lambda)| \leq \ln |\mu|(D)+M(D)|\lambda| \leq\left(\ln ^{+}|\mu|(D)+M(D)\right)(|\lambda|+1), \quad \lambda \in \mathbb{C}^{p} .
$$

If $\inf \{u(z): z \in D\}=u_{0}$, the definition of $V$ shows that

$$
V(\lambda)+\ln |\widehat{S}(\lambda)| \leq v(\lambda) \leq \sup _{z \in D} \operatorname{Re}\langle\lambda, z\rangle-u_{0} \leq\left(M(D)+u_{0}^{-}\right)(|\lambda|+1)
$$

for all $\lambda \in \mathbb{C}^{p}$. First, we assume that $\widehat{S}(0) \neq 0$. By (3.3) and (3.4), the assumptions of Lemma 6 are fulfilled for $V$ and $\ln |\widehat{S}|$. Consequently, there is a constant $A$ depending only on $D, S$, and $u$ and such that $V(\lambda) \leq A(|\lambda|+1), \lambda \in \mathbb{C}^{p}$. But if $\widehat{S}(0)=0$, we take $\lambda_{0}$ with $\left|\lambda_{0}\right|<1$ and $\widehat{S}\left(\lambda_{0}\right) \neq 0$, and then estimate the functions $V\left(\lambda+\lambda_{0}\right)+\ln \left|\widehat{S}\left(\lambda+\lambda_{0}\right)\right|$ and $\ln \left|\widehat{S}\left(\lambda+\lambda_{0}\right)\right|$, obtaining an estimate for $V\left(\lambda+\lambda_{0}\right)$. 
Now we estimate $V$ from below. Let $\sup \left\{u(z): z \in D_{m}\right\}=M^{\prime}$. Then $v(\lambda) \geq$ $\sup \left\{\operatorname{Re}\langle\lambda, z\rangle-u(z): z \in D_{m}\right\} \geq h_{m}(\lambda)-M^{\prime}, \lambda \in \mathbb{C}^{p}$. Taking (3.2) into account, we obtain $V(\lambda) \geq-M^{\prime}-\ln |\mu|(D)$. Thus, there exists a constant $B^{\prime}$ such that $|V(\lambda)| \leq$ $B^{\prime}(|\lambda|+1), \lambda \in \mathbb{C}^{p}$.

Lemma 7 is proved.

Proof. Using Lemmas 5-7, we can prove Theorem 1. In $\S 1$, the problem of spectral synthesis in the kernel of $M_{S}$ was reduced to the problem of completeness of the polynomials in the inductive limit of the spaces $P\left(v_{k}-\ln |\widehat{S}|\right)$. Next, by (3.1), it suffices to prove that the polynomials are complete in the inductive limit of the spaces $P\left(V_{k}\right)$. Put $w_{k}(\lambda)=\int_{\mathbb{C}^{p}} \alpha(\zeta-\lambda) V_{k}(\zeta) d m(\zeta)$. The first statement of Lemma 7 implies that the spaces $P\left(w_{k}\right)$ and $P\left(V_{k}\right)$ coincide, and it suffices to prove that the polynomials are complete in the inductive limit of the spaces $P\left(w_{k}\right)$.

We show that each $w_{k}$ satisfies the assumptions of Theorem 2. By the second statement of Lemma $7, w_{k}$ satisfies (R) with some constant $B$ and $s=\frac{1}{2}$. We estimate each $w_{k}$ from below. By assumption, $S$ is representable as in (1). Let $m$ be the smallest natural number such that $\operatorname{supp} \mu \subset D_{m}$ (see (4)). Take an arbitrary index $k>m$ and put $r_{k}=\sup \left\{u_{k}(z): z \in D_{k}\right\}$. The Young conjugates obey the estimate $v_{k}(\lambda) \geq$ $\sup \left\{\operatorname{Re}\langle\lambda, z\rangle-r_{k}: z \in D_{k}\right\}=h_{k}(\lambda)-r_{k}$. Together with (3.4), this shows that

$$
\frac{e^{v_{k}(\lambda)}}{|\widehat{S}(\lambda)|} \geq \frac{1}{|\mu|(D) e^{r_{k}}} e^{h_{k}(\lambda)-h_{m}(\lambda)}, \quad \lambda \in \mathbb{C}^{p} .
$$

Since $D_{s}+D\left(0, \frac{1}{s(s+1)}\right) \subset D_{s+1}$ for any $s, h_{s+1}(\lambda) \geq h_{s}(\lambda)+\frac{1}{s(s+1)}|\lambda|$ for all $\lambda \in \mathbb{C}^{p}$. Thus, from (3.5) we deduce that $v_{k}(\lambda)-\ln |\widehat{S}(\lambda)| \geq-\ln |\mu|(D)-r_{k}+|\lambda| /(m(m+1))$. By statement 1 of Lemma 7, the same is true for the regularizations: $V_{k}(\lambda) \geq-\ln |\mu|(D)-$ $r_{k}+|\lambda| /(m(m+1)), \lambda \in \mathbb{C}^{p}$. It remains to estimate the $w_{k}$ from above. Let $b_{k}=$ $\inf \left\{u_{k}(z): z \in D\right\}$. Then $v_{k}(\lambda) \leq \sup \left\{\operatorname{Re}\langle\lambda, z\rangle-b_{k}: z \in D\right\} \leq M(D)|\lambda|-b_{k} \leq$ $\left(M(D)+\left|b_{k}\right|\right)(|\lambda|+1)$, whence $V_{k}(\lambda)+\ln |\widehat{S}(\lambda)| \leq v_{k}(\lambda) \leq\left(M(D)+\left|b_{k}\right|\right)(|\lambda|+1)$. Assume first that $\widehat{S}(0) \neq 0$. We apply Lemma 6 to the functions $V_{k}$ and $\ln |\widehat{S}|$. By (3.2) and the last estimate, it follows that $\exp V_{k}$ is of the first order and finite type. By Lemma 7, the regularization $w_{k}$ admits the estimate $w_{k}(\lambda) \leq \Delta(|\lambda|+1), \lambda \in \mathbb{C}^{p}$. But if $\widehat{S}(0)=0$, we take $\lambda_{0},\left|\lambda_{0}\right|<1$, with $\widehat{S}\left(\lambda_{0}\right) \neq 0$ and apply Lemma 6 to the functions $V_{k}\left(\lambda+\lambda_{0}\right)$ and $\ln \left|\widehat{S}\left(\lambda+\lambda_{0}\right)\right|$. Thus, every $w_{k}$ with $k>m$ satisfies the assumptions of Theorem 2 with $s=\frac{1}{2}$ and some $B, \Delta$, and $\delta$. By that theorem the functions in $P\left(w_{k}\right)$ can be approximated by polynomials in the norm of $P\left(w_{k}+(2 p+1) \ln \left(1+|\lambda|^{2}\right)\right)$. By condition (3), the functions in $P\left(w_{k}\right)$ can be approximated by polynomials in the norm of $P\left(w_{k+2 p+1}\right)$. Consequently, the polynomials form a dense subset of the inductive limit $P(D, U)$.

Theorem 1 is proved.

\section{REFERENCES}

[1] L. Ehrenpreis, Fourier analysis in several complex variables, Pure Appl. Math., vol. 17, WileyIntersci. Publ., New York, 1970. MR.0285849 (44:3066)

[2] I. F. Krasichkov-Ternovskiǔ, A homogeneous convolution type equation on convex domains, Dokl. Akad. Nauk SSSR 197 (1971), no. 1, 29-31; English transl., Soviet Math. Dokl. 12 (1971), 396-398. MR0277729(43:3462)

[3] B. Malgrange, Existence et approximation des solutions des équations dérivées partielles et des équations de convolution, Ann. Inst. Fourier (Grenoble) 6 (1955-1956), 271-355. MR0086990 $(19: 280 \mathrm{a})$

[4] L. Ehrenpreis, Mean periodic function, Amer. J. Math. 77 (1955), 293-328. MR0070047(16:1122d)

[5] V. V. Napalkov, Convolution equations in multidimensional spaces, Nauka, Moscow, 1982. (Russian) MR0678923 (86g:46054) 
[6] R. S. Yulmukhametov, Homogeneous convolution equations, Dokl. Akad. Nauk SSSR 316 (1991), no. 2, 312-315; English transl., Soviet Math. Dokl. 43 (1991), no. 1, 101-103. MR.1100598 (92c:32005)

[7] O. V. Epifanov, Duality of a pair of spaces of analytic functions of bounded growth, Dokl. Akad. Nauk SSSR 319 (1991), no. 6, 1297-1300; English transl., Soviet Math. Dokl. 44 (1992), no. 1, 314-317. MR.1150105 (93d:46039)

[8] N. F. Abuzyarova and R. S. Yulmukhametov, Dual spaces of weighted spaces of analytic functions, Sibirsk. Mat. Zh. 42 (2001), no. 1, 3-17; English transl., Siberian Math. J. 42 (2001), no. 1, 1-14. MR 1830787 (2002c:46047)

[9] A. Grothendieck, Sur les espaces $(F)$ et $(D F)$, Summa Brasil. Math. 3 (1954), 57-123. MR0075542 $(17: 765 b)$

[10] A. S. Krivosheev and V. V. Napalkov, Complex analysis and convolution operators, Uspekhi Mat. Nauk 47 (1992), no. 6, 3-58; English transl., Russian Math. Surveys 47 (1992), no. 6, 1-56. MR 1209144 (94e:32003)

[11] N. Sibony, Approximation polynomiale pondérée dans un domaine d'holomorphie de $\mathbb{C}^{n}$, Ann. Inst. Fourier (Grenoble) 26 (1976), no. 2, 77-99. MR0430312 (55:3317)

[12] L. I. Ronkin, Introduction to the theory of entire functions of several variables, Nauka, Moscow, 1971; English transl., Transl. Math. Monogr., vol. 44, Amer. Math. Soc., Providence, RI, 1974. MR0320357(47:8896) MR0346175 (49:10901)

[13] I. F. Krasichkov-Ternovskiı̆, Estimates for a subharmonic difference of subharmonic functions. I, Mat. Sb. (N. S.) 102(144) (1977), no. 2, 216-247; English transl., Math. USSR-Sb. 31 (1977), no. 2, 191-218. MR0507987 (58:22602)

[14] L. Hörmander, An introduction to complex analysis in several variables, North-Holland Math. Library, vol. 7, North-Holland Publ. Co., Amsterdam, 1990. MR.1045639 (91a:32001)

[15] R. T. Rockafellar, Convex analysis, Princeton Math. Ser., vol. 28, Princeton Univ. Press, Princeton, NJ, 1970. MR0274683 (43:445)

Institute of Mathematics with Computing Centre, 112 Chernyshevsky Street, Ufa 450077, Russia

E-mail address: Yulmukhametov@mail.ru

Received 2/APR/2007

Translated by S. V. KISLYAKOV 